\title{
Monitoring treatment response in psoriasis: current perspectives on the clinical utility of reflectance confocal microscopy
}

This article was published in the following Dove Press journal:

Psoriasis: Targets and Therapy

20 February 2017

Number of times this article has been viewed

\author{
Marina Agozzino' \\ Cecilia Noal ${ }^{2}$ \\ Francesco Lacarrubba ${ }^{3}$ \\ Marco Ardigò ${ }^{4}$ \\ 'Dermatology Unit, Second University \\ of Naples, Naples, ${ }^{2}$ Dermatology \\ Unit, University of Trieste, Trieste, \\ ${ }^{3}$ Dermatology Clinic, University \\ of Catania, Catania, ${ }^{4} \mathrm{Clinical}$ \\ Dermatology Department, San \\ Gallicano Dermatological Institute, \\ Istituto di Ricovero e Cura a \\ Carattere Scientifico, Rome, Italy
}

\begin{abstract}
Reflectance confocal microscopy (RCM) evaluation of inflammatory skin diseases represents a relatively new technique that, during the past 5 years, has attracted increasing interest, with consequent progressive increment of publications in literature. The success of RCM is directly related to the high need for noninvasive techniques able to both reduce the number of skin biopsies and support clinical diagnosis and patient management. RCM helps to visualize microscopic descriptors of plaque psoriasis (PP) with good reproducibility between observers and a high grade of correspondence with histopathology. Several clinical tests are used for the therapeutic management of PP, but they are limited by subjective interpretation. Skin biopsy presents objective interpretation, but the procedure is invasive and not repeatable. RCM has been used not only for the evaluation of skin cancer or inflammatory skin diseases, but also for monitoring the efficacy of different treatments in PP. In this review, we present some examples of RCM applications in therapeutic psoriasis follow-up.
\end{abstract}

Keywords: reflectance confocal microscopy, psoriasis noninvasive follow-up, psoriasis monitoring

\section{Introduction}

Psoriasis is a complex and chronic inflammatory disease affecting a large number of patients all over the world. ${ }^{1}$ Treatments involve different approaches. Therapy includes topicals, phototherapy and other systemic drugs such as methotrexate, cyclosporine, acitretine and fumarates. ${ }^{2,3}$ Biological agents represent targeted molecular therapy, showing good efficacy and improving the quality of life of patients, with low risk of toxic effects. Currently, studies have been performed to test the safety and efficacy of new treatment options for psoriasis, eg, innovative biologic therapies. In the development and assessment of new therapeutic modalities, a clear definition of a patient's psoriasis severity is crucial. The impact of a therapy can be evaluated based on the changes of the severity score during and after treatment. For the evaluation of the disease severity and activity, several physical measures such as body surface area (BSA), psoriasis area and severity index (PASI) and other important techniques such as dermoscopy have been applied. ${ }^{4}$ However, the currently available psoriasis scoring systems have several limitations. ${ }^{4}$

For monitoring the histological effects of new treatment options, skin biopsy is inevitable. However, skin biopsy is not easily feasible due to its invasiveness and does not represent the perfect way of dynamically monitoring skin changes, due to the impossibility of evaluating the same area over time and the inflammatory effects
Clinical Dermatology Department, San Gallicano Dermatological Institute Istituto di Ricovero e Cura a Carattere Scientifico, Via Elio Chianesi 53, Rome $00 \mid 44$, Italy

Tel +390652666710 ext 6000

Fax +39065266 6158

Email marco.ardigo@ifo.gov.it 
occurring in the surrounding tissues during wound healing. In addition, a biopsy represents only a small sample of the lesion, which potentially results in sample error, and it is an invasive procedure for the patient with potential for scarring. In literature, it has already been demonstrated that the identification of the main diagnostic microscopic features characterizing plaque psoriasis (PP) is easily possible using in vivo reflectance confocal microscopy (RCM). ${ }^{5,6} \mathrm{RCM}$ examination of PP demonstrates different degrees of changes that are analyzed considering the different skin layers and can be used to overcome the limitations associated with biopsies.

Recent prospective studies demonstrated that RCM improves diagnostic accuracy and spares numerous unnecessary excisions, providing descriptive features of the most common inflammatory skin diseases. ${ }^{7-9}$ Concerning PP, RCM criteria and their histopathologic correlates have been successfully exploited. ${ }^{5,6}$ More recently, starting from the description of the confocal microscopic patterns, a multicentric study designed and executed under the supervision of the International Confocal Group (ICG) has been conducted with the aim of the identification of specific confocal features useful for the distinction between the 3 main groups of inflammatory skin diseases (spongiotic dermatitis, psoriasiform dermatitis and interface dermatitis). ${ }^{10}$ The study was conducted on a large number of cases affected by different superficial inflammatory skin diseases representing the different groups of entities. The defined collection of confocal features has been translated into an algorithmic method of analysis, confirming the potential of RCM for the diagnosis of and differential diagnosis between the different inflammatory skin disease groups. Moreover, due to the possibility of observation of detailed microscopic changes, RCM has been adopted for in vivo evaluation of therapeutic follow-up and disease progression. ${ }^{11,12}$ Recent data suggest the usefulness of RCM for the management of PP during topical and systemic treatments, demonstrating the possibility of detailed monitoring of microscopic changes during different therapies. ${ }^{13,14}$ In the following sections, we report on the state of the art in RCM and the therapeutic follow-up of PP.

\section{RCM for monitoring ultraviolet $B$ (UVB) phototherapy, topical treatment and systemic treatment}

RCM has been used for diagnosis improvement ${ }^{7-9}$ and treatment follow-up of inflammatory skin diseases. ${ }^{15-17}$ In literature, a good correlation between clinical symptoms, dermoscopy histological features and confocal imaging in inflammatory lesions has been described.
The first study that used RCM as a noninvasive tool to monitor therapeutic response of UVB phototherapy in patients with PP was conducted by Wolberink et al. ${ }^{18}$ This pilot study involved a limited number of patients $(n=6)$, and RCM imaging was performed before the narrow band UVB therapy, after 9 irradiations, at the end of the treatment and at 12 weeks after clinical clearance. A biopsy lesional skin examination was performed in 4 out of the 6 patients. In 2 patients, a biopsy of normal skin was taken. Results revealed a good correlation between clinical clearance, hematoxylin-eosin-stained histopathology and confocal parameters. Immunohistochemical staining (IHC) was also performed with monoclonal antibodies specific for cluster of differentiation (CD)31, CD3, filaggrin, Ki67, K16 and CD1a. Dermoscopy evaluation of PP lesions revealed the presence of oval red dots corresponding to blood overflow and enlargement of dermis capillaries in 2 of the 6 patients. Confocal and histological criteria considered were parakeratosis, orthokeratosis, epidermal inflammatory cells, atypical honeycomb pattern, papillomatosis (increased diameter of dermal papillae (DPs), upmigrated DPs and thin interpapillary spaces), number of papillary capillaries and capillary blood flow in DPs. DPs did not present any demarcating bright rim of basal cells (nonrimmed DPs) in 3 of the 6 patients. Results revealed a good correlation between clinical clearance, hematoxylin-eosin staining and confocal parameters. At baseline, dermoscopic evaluation revealed oval red dots corresponding to rapid blood flow and enlargement of dermal capillaries, especially in 2 patients. Because RCM is an in vivo imaging technique, dynamic micrometric and morphometric changes could be detected, especially blood flow. At RCM, dilated blood vessels were perpendicular to the face orientation, which corresponded to the roundish/oval red dots seen in dermoscopy.

After 9 irradiations, there was clinical improvement, with a reduction of red dots seen in dermoscopy. Parakeratosis, inflammatory cells and area of atypical keratinocytes decreased, as well as capillary blood flow. Clinical clearance corresponded to the normal/pigmented dermoscopic pattern. RCM imaging showed improvement in terms of cellular atypia and inflammatory cells, but acanthosis and papillomatosis were still present. RCM and histological evaluation were completely comparable to the nonlesional clinical skin parameters only after 12 weeks after clearance. Target lesions were all characterized by a regular honeycomb pattern, as well as minimal capillary blood flow with transverse orientation of blood vessel. During confocal evaluation, no dendritic cells were visualized despite their presence in IHC 
analysis. The authors hypothesized that the CD1a-positive dendritic cells observed in IHC may be nonactivated resident macrophages and only this type of dendrite could be visualized by confocal imaging. In this study, the presence of large clustered tortuous capillaries at baseline seemed to be prognostic of nonresponsiveness to UVB phototherapy, because 2 patients presenting these criteria did not present clinical or microscopic clearance at the end of treatment compared to those with small and solitary capillaries. In this way, capillary diameter was suggested by the authors to be prognostic for phototherapy outcome, but other studies are necessary in a major patient population.

Ardigò et $\mathrm{al}^{13}$ evaluated the in vivo microscopic changes in PP occurring during treatment with 2 distinct active compounds (aceclofenac and betamethasone) on 32 patients. This was the first comparative confocal and histological study using 2 different topical drugs in psoriasis. The authors developed a specific analysis of treatment effect pathways related to different mechanisms of action. Two lesions of PP taken from the same area or located symmetrically (left arm, right arm) were selected at baseline and assigned to the specific topical treatment: aceclofenac gel, which inhibits cyclooxygenase at inflammatory sites and suppresses prostaglandin E2 production by inflammatory cells (aceclofenac arm), and betamethasone cream, which acts on keratinocyte proliferation and strongly inhibits inflammatory cell activity (betamethasone arm). The RCM criteria defining PP used in different studies are summarized in Table 1.

Clinical and dermoscopy data collected did not show significant difference, except for faster steroid inflammatory reduction. On RCM, authors observed different response pathways for each treatment. Both agents were active in reducing overall skin thickness and spongiosis and in inducing normal keratinocyte maturation, as documented by partial reduction in parakeratosis. Interestingly, some RCM parameters showed different trends with different treatments. In fact, steroid cream seemed to negatively affect the metabolic activity of keratinocytes, as deduced by the observation of blurred keratinocyte borders in a large percentage of cases at the end of the study. On the other hand, only nonsignificant increase in blurred keratinocytes was detectable after aceclofenac treatment, with better outlined contours detectable at the end of the study in some cases. This observation could be related to the well-known "atrophogenic" effect induced by steroids that is seen on RCM as blurriness of keratinocyte contour, a sign of cellular metabolism being affected. Moreover, reduction in the presence of uplocated DPs, representing a sign of papillomatosis, was more evident in the aceclofenac-treated lesions. This can be interpreted as a better targeted activity of aceclofenac to reduce proinflammatory signal release from keratinocytes and inflammatory cells, with consequent regularization of keratinocyte proliferation

Table I RCM features of PP considered in different studies

\begin{tabular}{|c|c|}
\hline RCM criteria & Definition \\
\hline SC thickness & $\begin{array}{l}\text { Calculated by } z \text { vertical images stack lined from the top of the SC to the stratum granulosum. It is defined as } \\
\text { "thickened" when it is more than } 20 \mu \mathrm{m} \text { on the face or } 40 \mu \mathrm{m} \text { from other localization. }\end{array}$ \\
\hline Parakeratosis & $\begin{array}{l}\text { Presence of nucleated cells in the SC, visualized as bright nuclei in dark corneocytes. Nucleated corneocytes are } \\
\text { detected and numbered. }\end{array}$ \\
\hline Orthokeratosis & Presence of dark anucleated cells with indistinct cellular borders in SC \\
\hline Stratum granulosum thickness & Calculated by $z$ vertical images stack lined from the top of SC to the stratum granulosum \\
\hline Epidermal thickness & $\begin{array}{l}\text { Increased epidermal thickness calculated using vertical Viva Stack imaging starting immediately below the SC to } \\
\text { the DEJ. It is considered "thickened" when it is more than } 60 \mu \mathrm{m} \text { on the face and more than } 90 \mu \mathrm{m} \text { on other body } \\
\text { localization. }\end{array}$ \\
\hline Irregular honeycomb pattern & Atypical keratinocytes at the level of the epidermis with absence of polygonal regular keratinocyte shape. \\
\hline Spongiosis & Darker areas at the level of the stratum spinosum, increased interkeratinocyte spaces. \\
\hline Inflammatory cells in epidermis & $\begin{array}{l}\text { Presence of bright, round-to-polygonal cells in the epidermis. Microabscesses could be detected as groups of bright } \\
\text { polygonal/round cells. }\end{array}$ \\
\hline Papillomatosis & $\begin{array}{l}\text { Presence of increased diameter of DPs }(>80 \mu \mathrm{m}) \text {, upmigrated DPs (visible at the upper epidermis and } \\
\text { corresponding to interpapillary cristae elongation) and decrease of interpapillary spaces. }\end{array}$ \\
\hline Number of nonrimmed DPs & Presence of dark DPs not surrounded by bright ring of basal keratinocytes. \\
\hline $\begin{array}{l}\text { Dilated blood vessels in } \\
\text { papillary dermis }\end{array}$ & Round-to-canalicular dark structures with thin wall, inside DPs at of the level of papillary dermis. \\
\hline $\begin{array}{l}\text { Vascularization in the papillary } \\
\text { dermis }\end{array}$ & Blood flow was detected and counted in videos \\
\hline $\begin{array}{l}\text { Inflammatory cells in the } \\
\text { dermis }\end{array}$ & Presence of bright, round-to-polygonal cells in the dermis \\
\hline
\end{tabular}

Abbreviations: DEJ, dermo-epidermal junction; DP, dermal papilla; PP, plaque psoriasis; RCM, reflectance confocal microscopy; SC, stratum corneum. 
and no effect on keratinocyte metabolism. On the other hand, steroid was rapidly effective in reducing skin inflammation and clinical presentation of disease, but it inhibited keratinocyte proliferation more than acting on cell maturation. To confirm the overall "inhibitory effect" of the steroid, the authors observed a much lower recovery of "rimmed papillae" in respect of aceclofenac-treated lesions (Figure 1). Appearance of "rimmed DPs" is strictly related to constitutive production and release of melanin within basal keratinocytes. During the inflammatory process in psoriasis, mainly due to the inhibitory effect of tumor necrosis factor (TNF)- $\alpha$ on melanogenesis, basal keratinocytes contain less melanin than those in healthy skin (which frequently results in a hypopigmented area immediately after disease healing). During aceclofenac treatment, proinflammatory molecule inhibition may result in a prompter restart of melanin production. The authors suggested that the recovery of DPs correlated with a good therapeutic outcome, confirming RCM as a good noninvasive, in vivo therapeutic follow-up tool. This study demonstrated how RCM is useful to follow the microscopic changes during different topical PP treatments, allowing the differentiation of the treatment effect pathways of different products related to the different mechanisms of action.

Basaran et $\mathrm{al}^{19}$ described the therapy efficacy using RCM in 25 patients affected by PP. This was an investigator-blinded internal-controlled follow-up study. Patients were assigned to 1 of 3 treatment groups according to the clinical severity of PP: phototherapy, systemic treatment (methotrexate, acitretin) or topical treatment (moderately potent topical corticosteroid and calcipotriol). Patients were monitored during the 8-week treatment in a noninvasive manner. The aims of this analysis were to study the microscopic in vivo changes during psoriasis treatment and to compare the confocal data with the PASI score modifications. In each group, the confocal criteria were identified: parakeratosis, acanthosis, nonrimmed DPs, spongiosis, atypical honeycomb pattern, epidermal and dermal inflammatory cells, epidermal thickness, papillomatosis, as well as Munro's microabscesses, dermal capillaries' diameter and blood vessels in DPs. In this study, cells with dendritic morphology were described to be increased in patients who underwent phototherapy, suggesting migration of Langerhans cells out of the epidermis due to

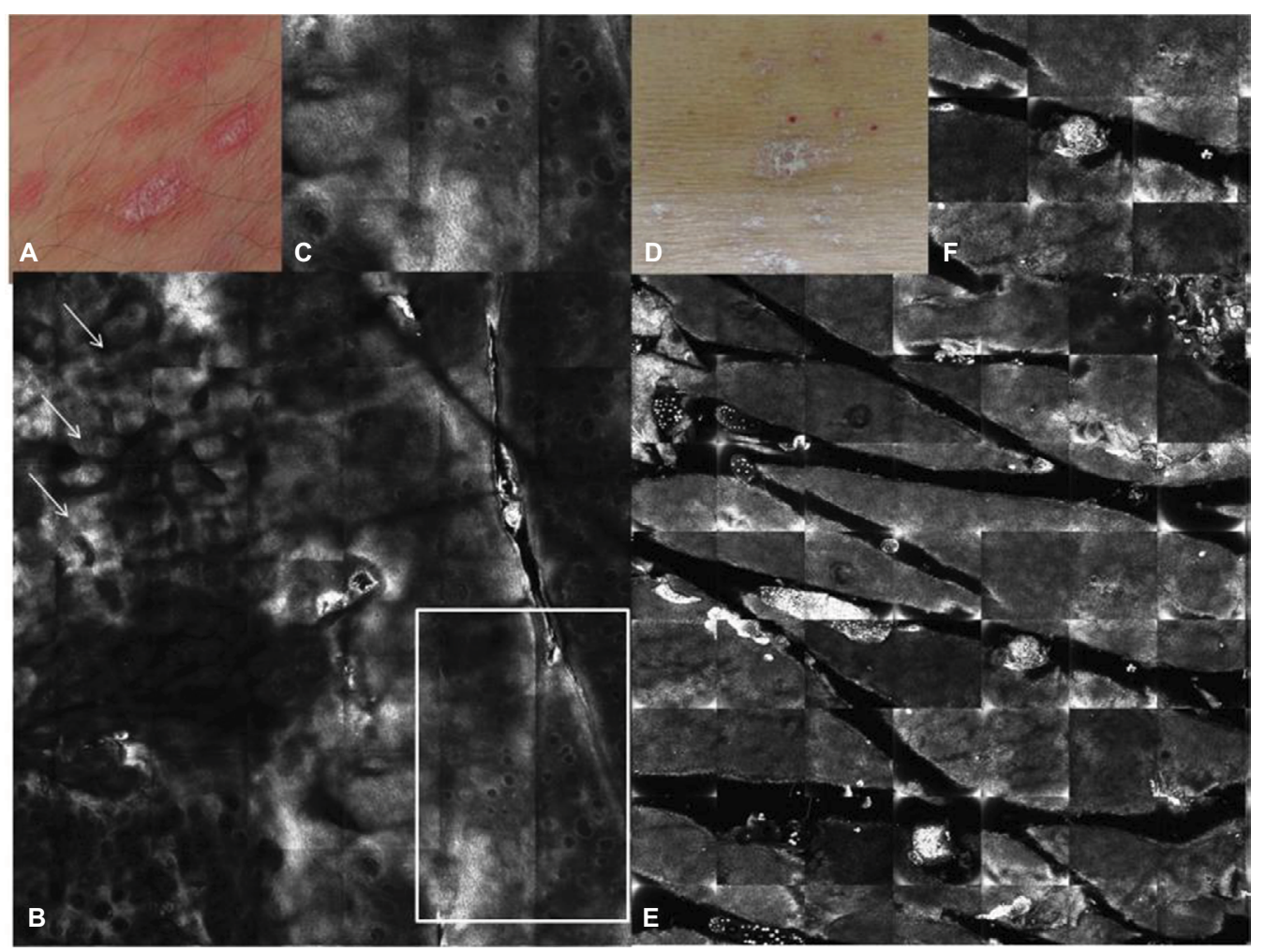

Figure I Therapeutic follow-up of plaque psoriasis.

Notes: Clinical images of PP at baseline (A) and after 12 weeks (D) of topical treatment (aceclofenac gel); corresponding RCM images taken at level of the epidermis from the same patient showing, at baseline, areas of hyperkeratosis (arrows) and superficial, dilated DPs without bright rimming and filled by dilated vessels (square) (B); normalization of stratum corneum and epidermis as sign of therapeutic response (E); RCM close up on the papillomatosis detected at baseline (C); recovery of the stratum corneum after 12 weeks of treatment $(\mathbf{F})$.

Abbreviations: DEJ, dermo-epidermal junction; DP, dermal papilla; PP, plaque psoriasis; RCM, reflectance confocal microscopy. 
this treatment. In this study, no biopsy was performed, so the nature of these dendritic cells was not defined by histological confirmation. Interestingly, Wolberink et al did not notice any dendritic cells at RCM during the evaluation, despite their presence on histology as confirmed by IHC.

Basaran et al made a comparative evaluation of confocal criteria and PASI score at baseline and at Weeks 4 and 8. RCM findings that correlated with changes in the PASI score were acanthosis, spongiosis, the number of irregular honeycomb-like sites, the presence of epidermal/dermal inflammatory cells, the number of focal microabscesses, the length of the papillary dermis and vascularization in the DPs. In particular, the authors observed that patients with high PASI score presented major areas of irregular honeycomb pattern and they were nonresponders to therapy. On the other hand, the number reduction of nonedge DPs appeared directly proportional to the clinical improvement, confirming that this criterion could be used as a parameter of response to the treatment. The limitation of this study was the lack of description of the correlation between each therapy and its confocal evaluation. Patients were divided in 3 groups, but the RCM criteria described were not correlated to a single therapeutic choice.

\section{RCM for monitoring biologic anti- TNF- $\alpha$ treatments}

Biological treatment represents a new approach to PP. Three categories of biological treatments are distinguished based on their modes of action: T-cell-modulating agents, inhibitors of TNF- $\alpha$ and inhibitors of interleukins 12, 13 and 17. In literature, only 1 study on the evaluation of utility of RCM in therapeutic follow-up of PP during treatment with adalimumab has been reported. Ardigò et $\mathrm{al}^{14}$ described the clinical symptoms, PASI score and confocal correlation during anti-TNF- $\alpha$ subcutaneous treatment. This multicenter study involved 48 patients with previous diagnosis of moderate-to-severe psoriasis. The target lesion was studied before starting biological treatment (T0), as well as after 4 (T1) and 8 (T2) weeks of treatment. Confocal images were correlated with clinical and dermoscopic results (Figures 2 and 3). In particular, the erythema, scaling and thickness of lesion were evaluated by clinicians on a scale of

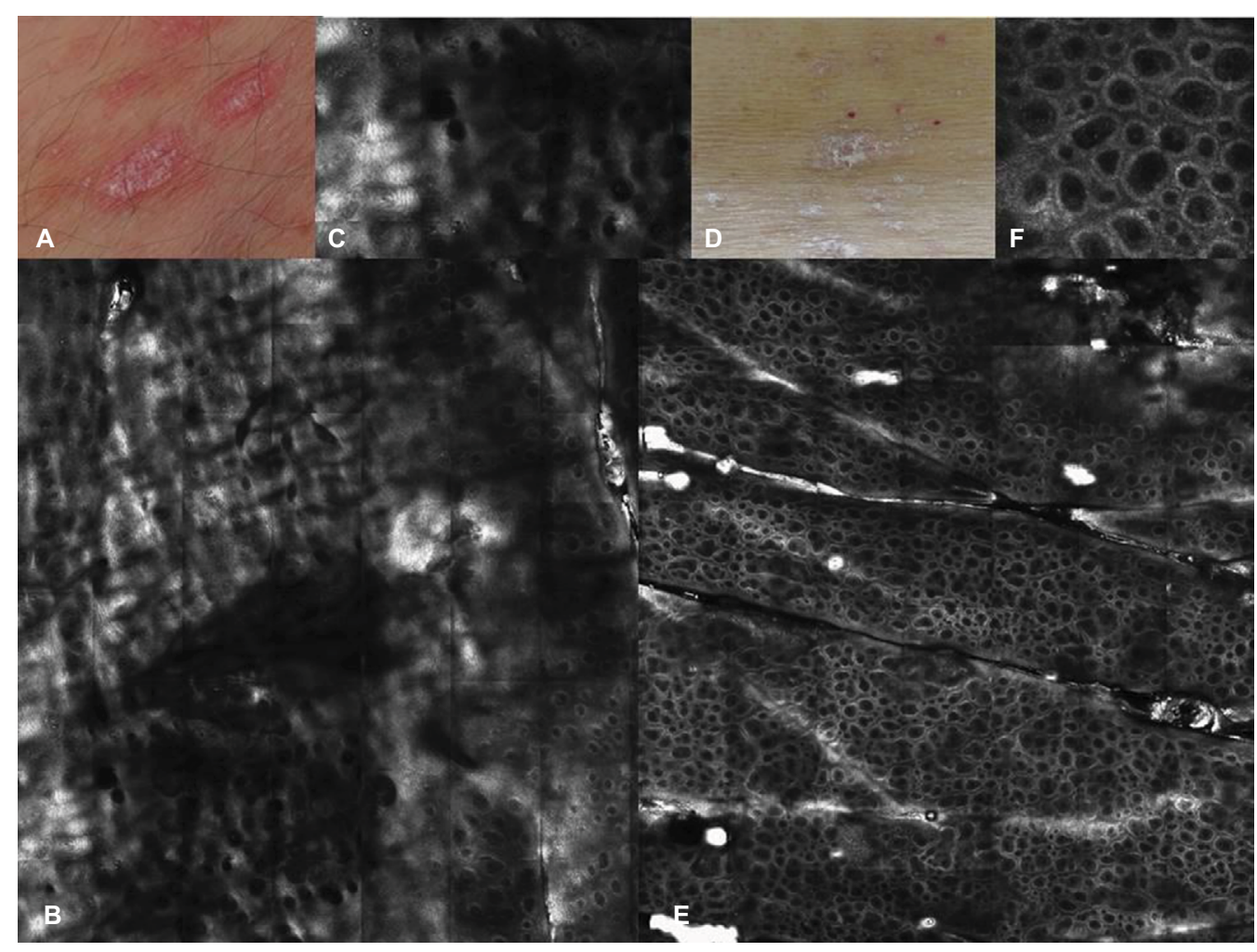

Figure 2 Clinical picture of PP on the trunk (A) at baseline and (D) after 8 weeks of systemic therapy (biologic anti-TNF- $\alpha$ treatment); (B) corresponding RCM taken at the baseline at the level of the epidermis, showing areas of hyperkeratosis and uplocated DPs as sign of papillomatosis; (E) after 8 weeks of treatment, RCM image showed a normal honeycombed pattern, with brighter and better-outlined keratinocyte contours and absence of uplocated DPs; (C and F) RCM close-up showing details of the uplocated DPs and the normal epidermis after treatment.

Abbreviations: DP, dermal papilla; PP, plaque psoriasis; RCM, reflectance confocal microscopy; TNF, tumor necrosis factor. 


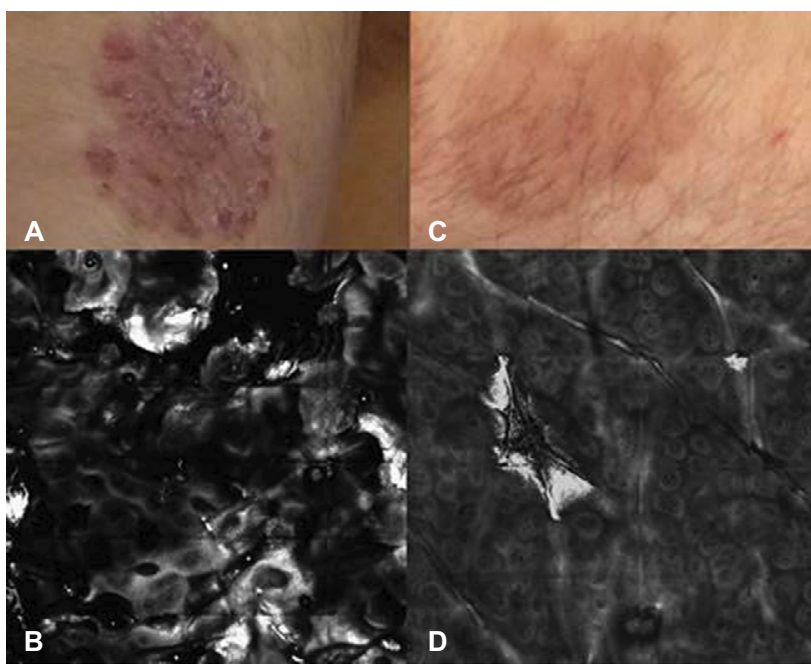

Figure 3 Therapeutic follow-up using RCM ather 8 weeks of anti-TNF- $\alpha$ therapy. Notes: Clinical images at baseline (A) and after 8 weeks (C). RCM perfomed at baseline shows hyperkeratosis and papillomatosis (B); after 8 weeks RCM shows disappearing of hyperkeratosis and reduction of DP size and density (D).

Abbreviations: DP, dermal papilla; RCM, reflectance confocal microscopy; TNF, tumor necrosis factor.

1-10 points. After 4 weeks of treatment with adalimumab, the PP lesions showed clinical improvement (60\%); and after 8 weeks, no patients presented skin disease signs. Parakeratosis and thickened epidermis had a progressive reduction between $\mathrm{T} 0$ and $\mathrm{T} 2$; however, the second criterion was statistically significant more than the first from $\mathrm{T} 0$ to $\mathrm{T} 2$ $(P=0.0001)$ than from T0 to T1 $(P=0.33)$. Acanthosis was reduced at $\mathrm{T} 2$, as well as the diameter of dilated vessels, clinical erythema and thickness of plaques. Infiltration of inflammatory cells into the epidermis decreased quickly at T0-T1 and T0-T2, but this was not statistically significant between T1 and T2. Moreover, dermal inflammatory cells were reduced between $\mathrm{T} 0$ and $\mathrm{T} 1$ and between $\mathrm{T} 0$ and $\mathrm{T} 2$. Indeed, the anti-inflammatory activity of adalimumab diminished the epidermal and superficial dermal inflammatory infiltration after 4 weeks of therapy. The early antiinflammatory action of anti-TNF- $\alpha$ was not correlated with the clinical improvement of erythema lesion background because there was a later reduction of dilated diameter vessels, the microscopic condition inducing psoriatic erythema. Regarding papillomatosis, dilated DPs and interpapillary spaces were decreased in comparison with the baseline, though upmigrated DPs were less identifiable between $\mathrm{T} 0$ and $\mathrm{T} 1$ than between $\mathrm{T} 0$ and $\mathrm{T} 2$. The reduction of $\mathrm{DP}$ diameter was observed only at 8 weeks. During treatment, DP rimming became identifiable compared at the baseline, with statistical significance between $\mathrm{T} 0$ and $\mathrm{T} 1(P=0.003)$

Table 2 Recent data on follow-up of PP using RCM

\begin{tabular}{|c|c|c|c|c|}
\hline Study & Authors & $\begin{array}{l}\text { Number of } \\
\text { patients }\end{array}$ & Methods & Results \\
\hline $\begin{array}{l}\text { Reflectance confocal } \\
\text { microscopy: an effective } \\
\text { tool for monitoring } \\
\text { ultraviolet B phototherapy } \\
\text { in psoriasis }\end{array}$ & $\begin{array}{l}\text { Wolberink } \\
\text { et al }\end{array}$ & 6 & $\begin{array}{l}\text { Confocal criteria (thick stratum corneum, } \\
\text { parakeratosis, intraepidermal inflammation, } \\
\text { acanthosis, papillomatosis, rimmed papillae, } \\
\text { dilated vessels, dermal inflammation) compared } \\
\text { to histology, dermoscopy and PASI }\end{array}$ & $\begin{array}{l}\text { Nonresponder patients present large } \\
\text { and tortuous capillaries at baseline of the } \\
\text { therapy. Capillaries show round diameter } \\
\text { with parallel or perpendicular orientation } \\
\text { corresponding to dermoscopy red dots }\end{array}$ \\
\hline $\begin{array}{l}\text { Psoriasis plaque test with } \\
\text { confocal microscopy: } \\
\text { evaluation of different } \\
\text { microscopic response } \\
\text { pathways in NSAID- and } \\
\text { steroid-treated lesions }\end{array}$ & $\begin{array}{l}\text { Ardigò } \\
\text { et } \mathrm{al}^{13}\end{array}$ & 32 & $\begin{array}{l}\text { Confocal criteria (thick stratum corneum, } \\
\text { parakeratosis, intraepidermal inflammation, } \\
\text { acanthosis, papillomatosis, rimmed papillae, } \\
\text { dilated vessels, dermal inflammation) compared } \\
\text { to dermoscopy }\end{array}$ & $\begin{array}{l}\text { RCM examination shows the different } \\
\text { effect pathways of } 2 \text { treatments. RCM is } \\
\text { able to distinguish the anti-inflammatory } \\
\text { and anti-TNF- } \alpha \text { activity of NSAID } \\
\text { (reduction of upmigrated DPs and } \\
\text { recovery of rimmed DPs) from the } \\
\text { "atrophogenic" effect induced by steroid } \\
\text { (blurred keratinocyte border) }\end{array}$ \\
\hline $\begin{array}{l}\text { Evaluation of the response } \\
\text { to treatment of psoriasis } \\
\text { vulgaris with reflectance } \\
\text { confocal microscopy }\end{array}$ & $\begin{array}{l}\text { Basaran } \\
\text { et } \text { al }^{18}\end{array}$ & 25 & $\begin{array}{l}\text { Confocal criteria (thick stratum corneum, } \\
\text { parakeratosis, intraepidermal inflammation, } \\
\text { acanthosis, papillomatosis, rimmed DPs, dilated } \\
\text { vessels, dermal inflammation) compared to } \\
\text { dermoscopy and PASI }\end{array}$ & $\begin{array}{l}\text { Number of rimmed DPs as a prognostic } \\
\text { positive value for treatment. Presence } \\
\text { of dendritic cells in patients treated by } \\
\text { phototherapy. Percentage of irregular } \\
\text { honeycomb pattern is related to high } \\
\text { PASI score and nonresponder patients }\end{array}$ \\
\hline $\begin{array}{l}\text { Reflectance confocal } \\
\text { microscopy for plaque } \\
\text { psoriasis therapeutic }\end{array}$ & $\begin{array}{l}\text { Ardigò } \\
\text { et al }{ }^{14}\end{array}$ & 48 & $\begin{array}{l}\text { Confocal criteria (thick stratum corneum, } \\
\text { parakeratosis, intraepidermal inflammation, } \\
\text { acanthosis, papillomatosis, rimmed DPs, dilated }\end{array}$ & $\begin{array}{l}\text { Early anti-inflammatory activity of } \\
\text { adalimumab: reduction of epidermal and } \\
\text { dermal inflammatory cell infiltration. }\end{array}$ \\
\hline $\begin{array}{l}\text { follow-up during an } \\
\text { anti-TNF- } \alpha \text { monoclonal } \\
\text { antibody: an observational } \\
\text { multicenter study }\end{array}$ & & & $\begin{array}{l}\text { blood vessels, dermal inflammation) compared } \\
\text { to dermoscopy and PASI }\end{array}$ & $\begin{array}{l}\text { Early reappearance of rimmed DPs as a } \\
\text { prognostic positive factor of response to } \\
\text { anti-TNF- } \alpha \text { therapy }\end{array}$ \\
\hline
\end{tabular}

Abbreviations: DP, dermal papilla; NSAID, nonsteroidal anti-inflammatory drugs; PASI, psoriasis area and severity index; PP, plaque psoriasis; RCM, reflectance confocal microscopy; TNF, tumor necrosis factor. 
and between $\mathrm{T} 0$ and $\mathrm{T} 2(P=0.001)$. In the second part of the study, the confocal criteria were divided into 3 different groups describing the following microscopic variations: the epidermal turnover, papillomatosis and the inflammation grade of infiltration. The presence of rimmed DPs was considered separately. These criteria were compared with the clinical scale evaluation and PASI scores, with results being statistically significant during follow-up. RCM examination revealed bright rims of DPs surrounding the DPs at T1, with statistical significance $(P<0.003)$. This could suggest the adalimumab inhibition of TNF- $\alpha$ inhibition of melanocyte activity and PP pathogenesis and the possibility that early reappearance of the dermo-epidermal junction (DEJ) pigmentation of keratinocytes could be a valuable indicator of early response to anti-TNF- $\alpha$ treatment in PP. ${ }^{20,21}$ All studies published on this topic are summarized in Table 2 .

\section{Conclusion}

Psoriasis is a chronic and relapsing systemic disease, so patients often require lifetime treatments. A spectrum of treatments is available to individualize patient therapy, including topicals (vitamin D3 analogs and corticosteroids), phototherapy with UVB, photochemotherapy (Psoralen + UVA), systemic treatments and biologics. Data on confocal microscopy of single inflammatory skin diseases, focusing mainly on confocal-histology correlation and therapeutic follow-up, have been published to date in the literature, demonstrating the effective practical application of RCM in real-life diagnostics and the differential diagnostic process of inflammatory skin diseases as well as therapeutic management. ${ }^{15,16}$ The in vivo imaging and the high confocal resolution permits the analysis of the severity of the disease and drives the choice of the right treatment at baseline as well as during therapeutic follow-up. RCM permits an objective, repeatable evaluation in psoriasis and other inflammatory skin disorders ${ }^{17}$ for monitoring therapeutic response pathways, reducing the number of biopsies for differential diagnosis or microscopic information. Further large and comparative studies on the possible use of RCM for PP management with different therapies, including treatment with different biologics, are needed in order to detect different early microscopic responses.

\section{Disclosure}

The authors report no conflicts of interest in this work.

\section{References}

1. Schon MP, Henning Boehncke W. Psoriasis. N Eng J Med. 2005;352: 1899-1912.
2. Murphy G, Reich K. In touch with psoriasis: topical treatments and current guidelines. J Eur Acad Dermatol Venereol. 2011;25(suppl 4):3-8.

3. Smith CH, Barker JNWN. Psoriasis and its management. BMJ. 2006; 333:380-384.

4. Naldi L. Scoring and monitoring the severity of psoriasis. What is the preferred method? What is the ideal method? Is PASI passé? Facts and controversies. Clin Dermatol. 2010;28(1):67-72.

5. Gonzalez S, Rajadhyaksha M, Rubinstein G, Anderson RR. Characterization of psoriasis in vivo by reflectance confocal microscopy. $J$ Med. 1999;30(5-6):337-356.

6. Ardigò M, Cota C, Berardesca V, González S. Concordance between in vivo reflectance confocal microscopy and histology in the evaluation of plaque psoriasis. J Am Acad Dermatol. 2009;23(6):660-667.

7. Agozzino M, Berardesca E, Donadio C, et al. Reflectance confocal microscopy features of seborrheicdermatitis for plaque psoriasis differentiation. Dermatology. 2014;229(3):215-221.

8. Ardigo M, Maliszewski I, Cota C, et al. Preliminary evaluation of in-vivo reflectance confocal microscope features of discoid lupus erythematosus. Br J Dermatol. 2007;156:1196-1203.

9. Astner S, Gonzalez E, Cheung A, Rius-Diaz F, Gonzalez S. Pilot study on the sensitivity and specificity of in vivo reflectance confocal microscopy in the diagnosis of allergic contact dermatitis. $J$ Am Acad Dermatol. 2005;53(6):986-992.

10. Ardigo M, Longo C, Gonzalez S; International Confocal Working Group Inflammatory Skin Diseases Project. Multicentre study on inflammatory skin diseases from The International Confocal Working Group: specific confocal microscopy features and an algorithmic method of diagnosis. Br J Dermatol. 2016;175(2):364-374.

11. Agozzino M, Donadio C, Franceschini C, Ardigò M. Therapeutic followup of lichen planopilaris using in vivo reflectance confocal microscopy: a case report. Skin Res Technol. 2015;21(3):380-383.

12. Ardigo M, Cameli N, Berardesca E, Gonzalez S. Characterization and evaluation of pigment distribution and response to therapy in melasmausing in vivo reflectance confocal microscopy: a preliminary study. J Eur Acad Dermatol Venereol. 2010;24(11):1296-1303.

13. Ardigò M, Agozzino M, Longo $C$, et al. Psoriasis plaque test with confocal microscopy: evaluation of different microscopic response pathways in NSAID and steroid treated lesions. Skin Res Technol. 2013; 19(4):417-423.

14. Ardigò M, Agozzino M, Longo C, et al. Reflectance confocal microscopy for plaque psoriasis therapeutic follow-up during an anti-TNF- $\alpha$ monoclonal antibody: an observational multicenter study. $J$ Eur Acad Dermatol Venereol. 2015;29(12):2363-2368.

15. Ardigo M, Agozzino M, Franceschini C, Lacarrubba F. Reflectance confocal microscopy algorithms for inflammatory and hair diseases. Dermatol Clin. 2016;34(4):487-496.

16. Agozzino M, Gonzalez S, Ardigò M. Reflectance confocal microscopy for inflammatory skin diseases. Actas Dermosifiliogr. 2016;107(8): 631-639.

17. Ardigò M, Prow T, Agozzino M, Soyer P, Berardesca E. Reflectance confocal microscopy for inflammatory skin diseases. G Ital Dermatol Venereol. 2015;150(5):565-573.

18. Wolberink EAW, van Erp PEJ, de Boer-van Huizen RT, van de Kerkhof PC, Gerritsen MJ. Reflectance confocal microscopy: an effective tool for monitoring ultraviolet B phototherapy in psoriasis. Br J Dermatol. 2012;167(2):396-403.

19. Basaran YK, Gürel MS, Erdemir AT, Turan E, Yurt N, Bağci IS. Evaluation of the response to treatment of psoriasis vulgaris with reflectance confocal microscopy. Skin Res Technol. 2015;21(1):18-24.

20. Martinez-Esparza M, Jimenez-Cervantes C, Solano F, Lozano JA, Garcia-Borron JC. Mechanisms of melanogenesis inhibition by tumor necrosis factor-alpha inB16/F10 mouse melanoma cells. Eur J Biochem. 1998;255(1):139-146.

21. Shang J, Eberle J, Gelen CC, et al. The role of nuclear factor-kappa B and melanogenesis in tumor necrosis factor-alpha-induced apoptosis of normal human melanocytes. Skin Pharmacol Appl Skin Physiol. 2002;15(5):321-329. 


\section{Publish your work in this journal}

Psoriasis: Targets and Therapy is international, peer-reviewed, open access journal focusing on psoriasis, nail psoriasis, psoriatic arthritis and related conditions, identification of therapeutic targets and the optimal use of integrated treatment interventions to achieve improved outcomes

and quality of life. Visit http://www.dovepress.com/testimonials.php to read real quotes from published authors.

Submit your manuscript here: https://www.dovepress.com/psoriasis-targets-and-therapy-journal 